\title{
EVALUACIÓN DE LA PRODUCTIVIDAD CON JORNADA DE 4-10 EN PROYECTOS DE CONSTRUCCIÓN EN SANTO DOMINGO, REPÚBLICA DOMINICANA
}

Evaluation of productivity with 4-10 work schedule on construction projects in Santo Domingo

\section{Tulio Rodríguez Fausto Fernely Moquete}

Resumen: El objetivo de esta investigación es medir la productividad laboral en proyectos de construcción en la ciudad de Santo Domingo, utilizando jornadas de 4 días y 10 horas propuesta por Thomas \& Horman (2006). El estudio se basa en el análisis de trabajadores divididos en cuatro brigadas de colocación de bloques de concreto y colocación de pisos de porcelanato equitativamente. Los resultados arrojados establecen que los niveles de productividad laboral son similares a la jornada actual, sin embargo los trabajadores les ha agradado más esta jornada, dadas las ventajas de organización que esta presenta. Concluimos que la jornada 4-10 puede ser un aporte más en mira de mejorar la productividad en el sector de la construcción en República Dominicana.

Palabras clave: productividad, construcción y jornada laboral. 
Abstract: This paper's objective is to measure productivity of labor in construction projects in Santo Domingo $\mathrm{RD}$, on a rolling 4/10 schedule proposed by Thomas \& Horman (2006). The methodology was to analyze workers in four brigades of bricks and floor tiles equitably. The results suggests that although the productivity is similar to the normal schedule of $5 / 8$, workers are more affined using this schedule, given the organizational advantages that represents. We conclude, that $4 / 10$ working schedule may be an input.

Keywords: productivity, construction and working hours.

\section{Introducción y justificación de la investigación}

La industria de la construcción es muy importante para la economía de la República Dominicana. El PIB de la construcción para el año 2013 fue de $4.2 \%$ siendo dentro del sector industrial el segundo más importante para la economía nacional. Genera entre 260,000 y 300,000 empleos directos que asciende a casi un millón de empleos indirectos lo que beneficia a todos los estratos sociales. El dinamismo de este sector se manifiesta en el efecto multiplicador que desata en el resto de la economía dominicana, tanto así que se utiliza como indicador de desarrollo económico (Banco Central, 2014).

No obstante, es una industria con niveles bajos de productividad. En una investigación realizada en la ciudad de Santo Domingo, utilizando la técnica de muestreo del trabajo se tomó una muestra de las diez actividades con mayor inversión en mano de obra en el sector de construcción dominicano. Se observó que el promedio de tiempo productivo en estas actividades es solo de un 31\%, mientras que el tiempo improductivo es de 56\% (Banco Central, 2014). 
Esto no es un problema de carácter exclusivo a nivel nacional. En Estados Unidos, desde el año 1964 hasta la actualidad, se ha observado que la productividad en los diversos sectores no agrícolas ha aumentado en un $100 \%$ a través de los años. Sin embargo, en el sector de la de construcción, aunque pueden observarse altas y bajas, es común que se experimente un decrecimiento de productividad en el tiempo (Forbes \& Ahmed, 2011).

A pesar de su importancia, la industria de la construcción es, incomprensiblemente, uno de los sectores que menor grado de desarrollo presenta en la mayoría de los países latinoamericanos, convirtiéndose en una actividad caracterizada por grandes deficiencias y falta de efectividad. Esto se traduce en una baja competitividad y coloca a las empresas constructoras en desventaja frente a otros mercados. (Botero, 2008).

En el sector de la construcción existe una línea muy delgada la obtención de ganancias y la generación de pérdidas. A pesar de que son muchos los factores que contribuyen al éxito de un proyecto de construcción, la productividad de la mano de obra es uno de los factores más importantes (Adrian, 2014).

La mejora de la productividad de la mano de obra de la construcción es un problema multifactorial, por lo que una única acción en el mismo, no puede brindar la solución a esta compleja problemática. Sin embargo, es necesario medir el resultado de cada acción para poder evaluar su aporte al todo. Este razonamiento se constituye en la premisa de la presente investigación, pues pretende evaluar el impacto del cambio en la jornada laboral semanal en la productividad de la construcción.

La jornada laboral a estudiar es organizada en 4 días a la semana, y 10 horas diarias, sin utilizar horas extras y descansando 3 días. No obstante, tras socializar esta investigación con alumnos y miembros del sector construcción nacional, expresaron sus dudas de forma casi generalizada. Estas dudas partían de tres perspectivas: 
el trabajador, el marco legal existente y la productividad. Estas dudas serán abordadas en el desarrollo de esta investigación.

La industria de la construcción dominicana está abierta a oportunidades de mejora (Senior \& Rodríguez, 2012) y con esta investigación se espera aportar al sistema de construcción de Santo Domingo otro tipo de jornada, con la cual pudieran ejecutar sus proyectos de una manera más eficiente y productiva, ahorrando tiempo y costos de producción.

\section{Objetivos de la investigación}

OBJETIVO GENERAL

Medir la productividad en proyectos de construcción en Santo Domingo, utilizando la jornada de 4 días y 10 horas.

OBjeTIVOS ESPECÍfICOS

- Comparar la jornada convencional de trabajo de 5 días a la semana, 8 horas de lunes a viernes, descansando 2 días, con la jornada de 4 días a la semana, y 10 horas diarias, sin utilizar horas extras y descansando 3 días.

- Determinar la productividad laboral, el rendimiento y los posibles ahorros en tiempo si se utiliza la jornada extendida 4-10.

\subsection{Antecedentes}

El principio 1 de un estudio realizado por $\mathrm{H}$. Randolph Thomas $\&$ Michael J. Horman en la revista Journal of construction engineering and management es: "Los contratistas deben considerar utilizar un horario de trabajo 4-10" (Thomas \& Horman, 2006). La base de datos de productividad para este informe contiene más de 125 proyectos. Hay más de 6000 días de trabajo implicando 
más de 300,000 horas de trabajo. No obstante, no existe dentro de la muestra ningún proyecto de República Dominicana.

\subsection{Marco legal}

La relación de empleadores y empleados es regulada en la nación por el Código de Trabajo de la República Dominicana. Este establece que la jornada normal es de 8 horas diarias y 44 horas semanales. A sabiendas de que según el artículo 162 del Código de Trabajo de la República Dominicana afirma lo siguiente:

Artículo 162. La Secretaria de Estado de Trabajo puede autorizar la distribución de las horas de trabajo en un periodo mayor de una semana, a condición de que la duración media del trabajo, calculada sobre el número de semanas consideradas, no exceda de cuarenta y cuatro horas por semana y de que en ningún caso las horas diarias de trabajo excedan de diez.

Como puede observarse en el artículo citado arriba, la implementación del tipo de jornada 4-10 en los proyectos de construcción en República Dominicana está dentro del contexto con base legal, debido a que la jornada laboral no sobrepasa las 10 horas diarias, ni las 44 horas semanales.

\section{Metodología de la investigación}

Para el desarrollo de la investigación se seleccionaron como partidas de estudio la colocación de bloques de concreto y colocación de pisos de porcelanato. Para el estudio de la productividad se utilizaron el muestreo de trabajo y el estudio de tiempo según la concepción teórica de Jim Adriam (Adrian \& Adrian, 1995).

Para el desarrollo de la investigación se seleccionó una muestra no probabilística de dos brigadas diferentes para cada partida de trabajo, es decir, 4 brigadas en total, para de esta manera comparar 
una brigada con otra realizando la misma actividad pero con jornadas diferentes. Las horas de observación de las mediciones fueron distribuidas equitativamente. Se trató de tomar la misma cantidad de mediciones tanto en la tarde con en la mañana, con intervalos de tiempo parecidos entre medición para poder lograr las cantidades mínimas requeridas por los métodos utilizados.

Para el muestreo de trabajo el período de duración de los estudios fue de dos semanas consecutivas y en el mismo se realizaron 384 observaciones por semana en cada tipo de jornada para ser consideradas estadísticamente válidas con un margen de error del 5\% y una confiabilidad del 95\%. Es decir, el tiempo de duración del estudio completo, incluyendo ambas jornadas será de 4 semanas por partida o 1 mes. Ambas partidas fueron realizadas al mismo tiempo con sus respectivas brigadas. Para el estudio de tiempos se utilizaron las mismas brigadas y fue dividido en dos tandas: una en la mañana y otra en la tarde; las mediciones se hicieron por actividades, cada actividad tuvo ocho ciclos.

Mediante el análisis de varianza (ANOVA) o la prueba Chi Cuadrado se evaluó el nivel de significancia estadística entre la diferencia de las medias de las siguientes variables:

- Tipo de jornada - Nivel de productividad

- Tipo de jornada - Nivel de rendimiento

- Brigada - Nivel de productividad

- Brigada - Nivel de rendimiento

Estos análisis fueron organizados según las partidas estudiadas. Para la realización de los mismos se utilizó el Software SPSS 15. 


\section{Análisis de resultados}

\section{COLOCACIÓN DE PISOS DE PORCELANATO}

\subsection{Muestreo de trabajo}

En las tablas a continuación se muestra el análisis de las variables Tipo de jornada - Nivel de productividad para las 3,072 observaciones realizadas.

Tabla N. ${ }^{\circ} 1$

Resumen del procesamiento de los casos

\begin{tabular}{|c|c|c|c|c|c|c|}
\hline \multirow{2}{*}{} & \multicolumn{6}{|c|}{ Casos } \\
\cline { 2 - 7 } & \multicolumn{2}{|c|}{ Válidos } & \multicolumn{2}{c|}{ Perdidos } & \multicolumn{2}{c|}{ Total } \\
\cline { 2 - 7 } & N & Porcentaje & N & Porcentaje & N & Porcentaje \\
\hline $\begin{array}{l}\text { Tipo de Jornada } * \\
\text { Tipo de Tiempo }\end{array}$ & 3072 & $100 \%$ & 0 & $0 \%$ & 3072 & $100 \%$ \\
\hline
\end{tabular}

Tabla N. ${ }^{\circ} 2$

Contingencia: Tipo de Jornada * Tipo de Tiempo

Recuento

\begin{tabular}{|c|c|c|c|c|}
\hline \multirow{2}{*}{$\begin{array}{c}\text { Tipo de } \\
\text { Jornada }\end{array}$} & \multicolumn{3}{|c|}{ Tipo de Tiempo } & \multirow{2}{*}{ Total } \\
\cline { 2 - 4 } & Productivo & Contributorio & $\begin{array}{c}\text { No } \\
\text { Contributorio }\end{array}$ & \\
\hline 5 días de 8 horas & 1017 & 354 & 165 & 1536 \\
\hline 4 días de 10 horas & 1059 & 310 & 167 & 1536 \\
\hline \hline Total & $\mathbf{2 0 7 6}$ & $\mathbf{6 6 4}$ & $\mathbf{3 3 2}$ & $\mathbf{3 0 7 2}$ \\
\hline
\end{tabular}


Tabla N. ${ }^{\circ} 3$

Pruebas de chi-cuadrado tipo de jornada

\begin{tabular}{|l|c|c|c|}
\hline & Valor & gl & $\begin{array}{c}\text { Sig. asintótica } \\
\text { (bilateral) }\end{array}$ \\
\hline Chi-cuadrado de Pearson & $3.777^{\mathrm{a}}$ & 2 & .151 \\
\hline Razón de verosimilitudes & 3.780 & 2 & .151 \\
\hline Asociación lineal por lineal & 1.128 & 1 & .288 \\
\hline N. de casos válidos & 3072 & & \\
\hline
\end{tabular}

a. 0 casillas $(0 \%)$ tienen una frecuencia esperada inferior a 5. La frecuencia mínima esperada es 166.00

Al obtener el valor de significancia de 0.151 que no es menor a 0.05 podemos establecer que no hay una diferencia estadísticamente significativa entre el tipo de jornada y los niveles de productividad. Por lo que se pudiera generalizar para estos datos que no existe una diferencia significativa entre los niveles de productividad según el tipo de jornada.

En las tablas a continuación se muestra la evaluación de las variables brigada y productividad.

Tabla N. ${ }^{\circ} 4$

Tabla de contingencia: Número de Brigada * Tipo de Tiempo Recuento

\begin{tabular}{|c|c|c|c|c|}
\hline \multirow{2}{*}{$\begin{array}{c}\text { Número de } \\
\text { Brigada }\end{array}$} & \multicolumn{3}{|c|}{ Tipo de Tiempo } & \multirow{2}{*}{ Total } \\
\cline { 2 - 4 } & Productivo & Contributorio & $\begin{array}{c}\text { No } \\
\text { Contributorio }\end{array}$ & \\
\hline 1 & 1008 & 358 & 170 & 1536 \\
\hline 2 & 1068 & 306 & 162 & 1536 \\
\hline \hline Total & $\mathbf{2 0 7 6}$ & $\mathbf{6 6 4}$ & $\mathbf{3 3 2}$ & $\mathbf{3 0 7 2}$ \\
\hline
\end{tabular}


Tabla N. ${ }^{\circ} 5$

Pruebas de chi-cuadrado número de jornada

\begin{tabular}{|l|c|c|c|}
\hline & Valor & gl & $\begin{array}{c}\text { Sig. asintótica } \\
\text { (bilateral) }\end{array}$ \\
\hline Chi-cuadrado de Pearson & $5.999^{a}$ & 2 & .050 \\
\hline Razón de verosimilitudes & 6.004 & 2 & .050 \\
\hline Asociación lineal por lineal & 3.260 & 1 & .071 \\
\hline N. de casos válidos & 3072 & & \\
\hline
\end{tabular}

a. 0 casillas $(0 \%)$ tienen una frecuencia esperada inferior a 5. La frecuencia mínima esperada es 166.00

$\mathrm{Al}$ obtener el valor de significancia de 0.05 que no es menor a 0.05 podemos establecer que no hay una diferencia estadísticamente significativa entre las 2 brigadas y los niveles de productividad.

\subsection{Estudio de Tiempo}

En las tablas a continuación se muestra el análisis de las variables Tipo de Jornada - Nivel de rendimiento para las 288 observaciones realizadas.

\section{Tabla N. ${ }^{\circ} 6$}

Análisis de las variables tipo de jornada - nivel de rendimiento Rendimiento $\mathrm{m}^{2} /$ hora

\begin{tabular}{|c|c|c|c|c|c|c|c|c|}
\hline & \multirow[b]{2}{*}{$\mathbf{N}$} & \multirow[b]{2}{*}{ Media } & \multirow[b]{2}{*}{$\begin{array}{c}\text { Desviación } \\
\text { típica }\end{array}$} & \multirow[b]{2}{*}{$\begin{array}{l}\text { Error } \\
\text { típico } \\
\end{array}$} & \multicolumn{2}{|c|}{$\begin{array}{c}\text { Intervalo de confianza } \\
\text { para la media al } 95 \%\end{array}$} & \multirow[b]{2}{*}{ Mínimo } & \multirow[b]{2}{*}{ Máximo } \\
\hline & & & & & $\begin{array}{l}\text { Límite } \\
\text { inferior }\end{array}$ & $\begin{array}{c}\text { Límite } \\
\text { superior }\end{array}$ & & \\
\hline $5-8$ & 160 & 6.4908 & 1.09797 & .08680 & 6.3194 & 6.6622 & 4.04 & 10.25 \\
\hline 4- 10 & 128 & 6.0454 & .76492 & .06761 & 5.9116 & 6.1792 & 3.92 & 8.56 \\
\hline Total & 288 & 6.2928 & .98790 & .05821 & 6.1783 & 6.4074 & 3.92 & 10.25 \\
\hline
\end{tabular}


Tabla N. ${ }^{\circ} 7$ - ANOVA 1

Rendimiento $\mathrm{m}^{2} /$ hora

\begin{tabular}{|l|c|c|c|c|c|}
\hline & $\begin{array}{c}\text { Suma de } \\
\text { cuadrados }\end{array}$ & gl & $\begin{array}{c}\text { Media } \\
\text { cuadrática }\end{array}$ & F & Sig. \\
\hline Inter-grupos & 14.108 & 1 & 14.108 & 15.170 & .000 \\
\hline Intra-grupos & 265.989 & 286 & .930 & & \\
\hline Total & 280.098 & 287 & & & \\
\hline
\end{tabular}

Se puede observar que el valor promedio de rendimiento de la jornada 5-8 es mayor al valor promedio de la jornada 4-10 (6.49 $>6.04 \mathrm{~m}^{2} /$ hora) y esta diferencia es estadísticamente significativa. Es decir que se puede generalizar que para estos datos la jornada 5-8 tuvo mayor rendimiento promedio.

En las tablas a continuación se muestra la evaluación de las variables brigada y rendimiento.

\section{Tabla N. ${ }^{\circ} 8$}

Análisis de las variables brigada y rendimiento

Rendimiento $\mathrm{m}^{2} /$ hora

\begin{tabular}{|c|c|c|c|c|c|c|c|c|}
\hline & \multirow[b]{2}{*}{$\mathbf{N}$} & \multirow[b]{2}{*}{ Media } & \multirow[b]{2}{*}{$\begin{array}{c}\text { Desviación } \\
\text { típica }\end{array}$} & \multirow[b]{2}{*}{$\begin{array}{l}\text { Error } \\
\text { típico }\end{array}$} & \multicolumn{2}{|c|}{$\begin{array}{c}\text { Intervalo de confianza } \\
\text { para la media al } 95 \%\end{array}$} & \multirow[b]{2}{*}{ Mínimo } & \multirow[b]{2}{*}{ Máximo } \\
\hline & & & & & $\begin{array}{l}\text { Límite } \\
\text { inferior }\end{array}$ & $\begin{array}{c}\text { Límite } \\
\text { superior }\end{array}$ & & \\
\hline 1 & 144 & 6.3402 & 1.00318 & .08360 & 6.1750 & 6.5055 & 3.96 & 10.25 \\
\hline 2 & 144 & 6.2455 & .97357 & .08113 & 6.0851 & 6.4059 & 3.92 & 9.19 \\
\hline Total & 288 & 6.2928 & .98790 & .05821 & 6.1783 & 6.4074 & 3.92 & 10.25 \\
\hline
\end{tabular}


Tabla N. ${ }^{\circ} 9$ - ANOVA 2

Rendimiento $\mathrm{m}^{2} /$ hora

\begin{tabular}{|l|c|c|c|c|c|}
\hline & $\begin{array}{c}\text { Suma de } \\
\text { cuadrados }\end{array}$ & g1 & $\begin{array}{c}\text { Media } \\
\text { cuadrática }\end{array}$ & F & Sig. \\
\hline Inter-grupos & .646 & 1 & .646 & .661 & .417 \\
\hline Intra-grupos & 279.452 & 286 & .977 & & \\
\hline Total & 280.098 & 287 & & & \\
\hline
\end{tabular}

Al obtener el valor de significancia de 0.417 que no es menor a 0.05 podemos establecer que no hay una diferencia estadísticamente significativa entre las 2 brigadas y el rendimiento.

\section{COLOCACIÓN DE BLOQUES}

\subsection{Muestreo de trabajo}

En las tablas a continuación se muestra el análisis de las variables Tipo de Jornada-Nivel de productividad para las 3,072 observaciones realizadas.

Tabla N. ${ }^{\circ} 10$

Resumen del procesamiento de los casos

\begin{tabular}{|c|c|c|c|c|c|c|}
\hline \multirow{2}{*}{} & \multicolumn{6}{|c|}{ Casos } \\
\cline { 2 - 7 } & \multicolumn{2}{|c|}{ Válidos } & \multicolumn{2}{c|}{ Perdidos } & \multicolumn{2}{c|}{ Total } \\
\cline { 2 - 7 } & N & Porcentaje & N & Porcentaje & N & Porcentaje \\
\hline $\begin{array}{l}\text { Tipo de Jornada * } \\
\text { Tipo de Tiempo }\end{array}$ & 3072 & $100 \%$ & 0 & $0 \%$ & 3072 & $100 \%$ \\
\hline
\end{tabular}


Tabla N. ${ }^{\circ} 11$

Contingencia: Tipo de Jornada $*$ Tipo de Tiempo

Recuento

\begin{tabular}{|c|c|c|c|c|}
\hline \multirow{2}{*}{$\begin{array}{c}\text { Tipo de } \\
\text { Jornada }\end{array}$} & \multicolumn{3}{|c|}{ Tipo de Tiempo } & \multirow{2}{*}{ Total } \\
\cline { 2 - 5 } & Productivo & Contributorio & $\begin{array}{c}\text { No } \\
\text { Contributorio }\end{array}$ & \\
\hline 5 días de 8 horas & 1031 & 313 & 192 & 1536 \\
\hline 4 días de 10 horas & 1001 & 421 & 114 & 1536 \\
\hline \hline Total & $\mathbf{2 0 3 2}$ & $\mathbf{7 3 4}$ & $\mathbf{3 0 6}$ & $\mathbf{3 0 7 2}$ \\
\hline
\end{tabular}

Tabla N. ${ }^{\circ} 12$

Pruebas de chi-cuadrado colocación de bloques

\begin{tabular}{|l|c|c|c|}
\hline & Valor & gl & $\begin{array}{c}\text { Sig. asintótica } \\
\text { (bilateral) }\end{array}$ \\
\hline Chi-cuadrado de Pearson & $36.216^{\mathrm{a}}$ & 2 & .000 \\
\hline Razón de verosimilitudes & 36.495 & 2 & .000 \\
\hline Asociación lineal por lineal & 1.683 & 1 & .194 \\
\hline N. de casos válidos & 3072 & & \\
\hline
\end{tabular}

a. 0 casillas $(0 \%)$ tienen una frecuencia esperada inferior a 5 . La frecuencia mínima esperada es 153.00

Al obtener el valor de significancia de 0.000 que es menor a 0.05 podemos establecer que hay una diferencia estadísticamente significativa entre el tipo de jornada y los niveles de productividad. Donde se puede establecer que en la jornada 4-10 existe una disminución de los tiempos no contributorios.

En las tablas a continuación se muestra la evaluación de las variables brigada y productividad. 
Evaluación de la productividad con jornada de 4-10 en proyectos de construcción en Santo Domingo, República Dominicana

Tabla N. ${ }^{\circ} 13$

Contingencia: Número de Brigada * Tipo de Tiempo

Recuento

\begin{tabular}{|c|c|c|c|c|}
\hline \multirow{2}{*}{$\begin{array}{c}\text { Número de } \\
\text { Brigada }\end{array}$} & \multicolumn{3}{|c|}{ Tipo de Tiempo } & \multirow{2}{*}{ Total } \\
\cline { 2 - 4 } & Productivo & Contributorio & $\begin{array}{c}\text { No } \\
\text { Contributorio }\end{array}$ & \\
\hline 1 & 1097 & 324 & 115 & 1536 \\
\hline 2 & 935 & 410 & 191 & 1536 \\
\hline \hline Total & $\mathbf{2 0 3 2}$ & $\mathbf{7 3 4}$ & $\mathbf{3 0 6}$ & $\mathbf{3 0 7 2}$ \\
\hline
\end{tabular}

Tabla N. ${ }^{\circ} 14$

Pruebas de chi-cuadrado número de productividad

\begin{tabular}{|l|c|c|c|}
\hline & Valor & gl & $\begin{array}{c}\text { Sig. asintótica } \\
\text { (bilateral) }\end{array}$ \\
\hline Chi-cuadrado de Pearson & $41.867^{\mathrm{a}}$ & 2 & .000 \\
\hline Razón de verosimilitudes & 42.103 & 2 & .000 \\
\hline Asociación lineal por lineal & 41.385 & 1 & .000 \\
\hline N. de casos válidos & 3072 & & \\
\hline
\end{tabular}

a. 0 casillas $(0 \%)$ tienen una frecuencia esperada inferior a 5. La frecuencia mínima esperada es 166.00

Al obtener el valor de significancia de 0.000 que es menor a 0.05 podemos establecer que hay una diferencia estadísticamente significativa entre las 2 brigadas y los niveles de productividad.

\subsection{Estudio de Tiempos}

En las tablas a continuación se muestra el análisis de las variables Tipo de Jornada-Nivel de rendimiento para las 288 observaciones realizadas. 
Tabla N. ${ }^{\circ} 15$

Análisis de las variables tipo de jornada - nivel de rendimiento Rendimiento $\mathrm{m}^{2} /$ hora

\begin{tabular}{|c|c|c|c|c|c|c|c|c|}
\hline & \multirow[b]{2}{*}{$\mathbf{N}$} & \multirow[b]{2}{*}{ Media } & \multirow[b]{2}{*}{$\begin{array}{c}\text { Desviación } \\
\text { típica }\end{array}$} & \multirow[b]{2}{*}{$\begin{array}{l}\text { Error } \\
\text { típico }\end{array}$} & \multicolumn{2}{|c|}{$\begin{array}{c}\text { Intervalo de confianza } \\
\text { para la media al } 95 \%\end{array}$} & \multirow[b]{2}{*}{ Mínimo } & \multirow[b]{2}{*}{ Máximo } \\
\hline & & & & & $\begin{array}{l}\text { Límite } \\
\text { inferior }\end{array}$ & $\begin{array}{c}\text { Límite } \\
\text { superior }\end{array}$ & & \\
\hline $5-8$ & 160 & 3.7494 & .57702 & .04562 & 3.6593 & 3.8395 & 2.50 & 5.41 \\
\hline $4-10$ & 128 & 4.0417 & .64886 & .05735 & 3.9282 & 4.1552 & 2.70 & 6.15 \\
\hline Total & 288 & 3.8793 & .62604 & .03689 & 3.8067 & 3.9519 & 2.50 & 6.15 \\
\hline
\end{tabular}

Tabla N. ${ }^{\circ} 16$ - ANOVA 3

Rendimiento $\mathrm{m}^{2} /$ hora

\begin{tabular}{|l|c|c|c|c|c|}
\hline & $\begin{array}{c}\text { Suma de } \\
\text { cuadrados }\end{array}$ & g1 & $\begin{array}{c}\text { Media } \\
\text { cuadrática }\end{array}$ & F & Sig. \\
\hline Inter-grupos & 6.075 & 1 & 6.075 & 16.328 & .000 \\
\hline Intra-grupos & 106.409 & 286 & .372 & & \\
\hline \hline Total & 112.484 & 287 & & & \\
\hline
\end{tabular}

Se puede observar que el valor promedio de rendimiento de la jornada 4-10 es mayor al valor promedio de la jornada 5-8 (4.04 $>3.75 \mathrm{~m}^{2} /$ hora) y esta diferencia es estadísticamente significativa.

En las tablas a continuación se muestra la evaluación de las variables brigada y rendimiento. 
Tabla N. ${ }^{\circ} 17$

Análisis de las variables brigada y rendimiento

Rendimiento $\mathrm{m}^{2} /$ hora

\begin{tabular}{|c|c|c|c|c|c|c|c|c|}
\hline & \multirow[b]{2}{*}{$\mathbf{N}$} & \multirow[b]{2}{*}{ Media } & \multirow[b]{2}{*}{$\begin{array}{c}\text { Desviación } \\
\text { típica }\end{array}$} & \multirow[b]{2}{*}{$\begin{array}{l}\text { Error } \\
\text { típico }\end{array}$} & \multicolumn{2}{|c|}{$\begin{array}{c}\text { Intervalo de confianza } \\
\text { para la media al } 95 \% \\
\end{array}$} & \multirow[b]{2}{*}{ Mínimo } & \multirow[b]{2}{*}{ Máximo } \\
\hline & & & & & $\begin{array}{l}\text { Límite } \\
\text { inferior }\end{array}$ & $\begin{array}{c}\text { Límite } \\
\text { superior }\end{array}$ & & \\
\hline 1 & 144 & 3.9413 & 69299 & .05775 & 3.8272 & 4.0555 & 2.50 & 6.15 \\
\hline 2 & 144 & 3.8174 & .54647 & .04554 & 3.7273 & 3.9074 & 2.59 & 5.41 \\
\hline Total & 288 & 3.8793 & .62604 & .03689 & 3.8067 & 3.9519 & 2.50 & 6.15 \\
\hline
\end{tabular}

Tabla N. ${ }^{\circ} 18$ - ANOVA 4

Rendimiento $\mathrm{m}^{2} /$ hora

\begin{tabular}{|l|c|c|c|c|c|}
\hline & $\begin{array}{c}\text { Suma de } \\
\text { cuadrados }\end{array}$ & gl & $\begin{array}{c}\text { Media } \\
\text { cuadrática }\end{array}$ & F & Sig. \\
\hline Inter-grupos & 1.106 & 1 & 1.106 & 2.841 & .093 \\
\hline Intra-grupos & 111.377 & 286 & .389 & & \\
\hline \hline Total & 112.484 & 287 & & & \\
\hline
\end{tabular}

Al obtener el valor de significancia de 0.093 que no es menor a 0.05 podemos establecer que no hay una diferencia estadísticamente significativa entre las 2 brigadas y el rendimiento.

\section{Conclusiones}

En el desarrollo de estas mediciones, se le planteó a los trabajadores la implementación de la jornada extendida 4-10 con tal de escuchar su pensar, pero estos reaccionaron de una manera incómoda, debido a que tendrían que llegar a la obra más temprano y se irían más tarde (la jornada era de 7:00 a.m. a 6 p.m. con una hora de almuerzo). 
No obstante, su percepción cambió cuando se le explicó que en la semana ganarían lo mismo, ya que las dos horas extras de los 4 días sería como trabajar 5 días. Los trabajadores al terminar el estudio expresaron su alta satisfacción con poder disponer de un día adicional a la semana para ellos, manteniendo su mismo nivel de ingreso. Inclusive en caso de que fuera necesario laborar horas extras el viernes, todavía disponían del fin de semana.

$\mathrm{Al}$ analizar los resultados de la variable tipo de jornada y nivel de productividad, medida por el método de estudio de tiempo en ningún caso resultó desfavorable la aplicación de la jornada 4-10 y en el caso de los de la colocación de los bloques mejoró.

Los resultados de los rendimientos fueron disímiles, pues para el caso de la colocación de pisos resultó mejor la jornada 5-8, pero para los bloques resultó mejor la 4-10. En virtud de lo dicho, debe establecerse una generalización de mejora para ninguna de los dos tipos de jornadas.

Al finalizar pude desmontarse la idea de que la jornada 4-10 no funciona en la República Dominicana. Las tres perspectivas que generan dudas han sido despejadas y abordadas. Los trabajadores se sintieron más a gusto con más esta jornada; el marco legal lo permite y los niveles de productividad son similares a la jornada actual.

Los constructores pueden considerar esta nueva opción de organizar su trabajo, la cual le permite tener ciertas ventajas:

- Disponer de un día adicional libre, el cual puede ser utilizado para tareas auxiliares como limpieza, recepción de materiales, pagos, entre otros sin interrumpir el ciclo normal de trabajo y con la ventaja que es un día laborable donde los suplidores y los comercios en sentido general está laborando.

- Disponer tres días libres a la semana que en un proyecto de altos requerimientos en cuanto a la fecha de entrega permitiría 

de construcción en Santo Domingo, República Dominicana

colocar otra brigada durante estos días y de esa manera tener doble turno semanal, sin tener que recurrir a los costos y riesgo que implica hacer esto utilizando el horario nocturno.

La jornada 4-10 no es "la solución" a todos los problemas de productividad de la industria de la construcción dominicana. En este mismo estudio de manera colateral se pudo observar:

- Los porcentajes de tiempo contributivo (TC) son muy significativos, por lo cual mientras mejor capacitados estén los trabajadores más rápido podría ser el desarrollo del trabajo, debido a que se invierte mucho tiempo explicándoles los procesos a los mismos.

- Además la planificación en las órdenes de materiales por parte del personal administrativo de los proyectos debe de ser más efectiva, porque muchos de los tiempos no contributivos (TNC) se debieron a la falta de material en obra al momento en que se agotaban.

No obstante, la jornada 4-10 puede ser un aporte más en mira de la mejora de la misma.

\section{Bibliografía}

Adrian, J. \& Adrian, D. (1995). Total Productivity and Quality Management for Construction. Illinois: Stipes Publishing L.L.C.

Adrian, J. (2014). Increasing job site productivity. World of concrete. Las Vegas: Hanley Wood Exhibitions.

Banco Central de la República Dominicana (2014). Producto Interno Bruto (PIB) por sectores de origen, a precios corrientes $y$ año de referencia 1991, anual. Recuperado de: http://www.bancentral. gov.do/estadisticas_economicas/real/ 
Botero, L. F. \& Álvarez, M. E. (junio de 2005). Last planner, un avance en la planificación y control de proyectos de construcción. Ingeniería \& desarrollo, 17, 148-159.

Botero, L. F. (2008). Construcción de edificaciones: aspectos administrativos. Medellin: Fondo Editorial Universidad EAFIT.

Campero, M. \& Alarcón, L. F. (2014). Administración de Proyectos Civiles (3ra edición ed.). Santiago, Santiago de Chile: Ediciones Universidad Católica de Chile.

Forbes, L. \& Ahmed, S. (2011). Modern Construction: Lean Proyect Delivery and integraded Practices. New York: CRC Press.

Gonzalez, N. \& Castillo, F. (2007). Analisis y pptimización del rendimiento y la productividad en la mano de obra para proyectos de edificaciones en la ciudad de Santo Domingo. Santo Domingo: Instituto Tecnológico de Santo Domingo.

Martínez, G. \& Pellicer, E. (2007). Organización y gestión de proyectos y obras. Madrid: McGraw-Hill/Interamericana de España.

Rojas Lopez, M. (2008). Gerencia de la construcción. Bogotá: Ecoe Ediciones.

Senior, B. A. \& Rodriguez, T. A. (2012). Analysing Barriers to Construction Productivity Improvement in the Dominican Republic. 20th Annual conference of the IGLC (pp. 701-710). San Diego, CA: Montezuma Publishing.

Serpell, A. (2002). Administración de operaciones. Mexico D. F.: Alfaomega.

Thomas, H. R. \& Horman, M. J. (enero de 2006). Fundamental Principles of Workforce Management. Journal of construction engineering and management, 132(1), 97-104.

Truyols, S., Saiz, J. M. \& García, D. (2006). Economía y organización de empresas para Arquitectura Técnica (Vol. 8). Madrid: Ediciones FIEC. 


\section{Tulio A. Rodríguez}

Es profesor del Instituto Tecnológico de Santo Domingo (INTEC). Enfocado en gestión de proyectos, administración estratégica, investigación en ingeniería civil y Construcción Lean. Posee una maestría de administración de la construcción del INTEC, una en Gerencia y Productividad de UNAPEC y un doctorado en educación de la universidad de Murcia. Director Facultativo del Parque Eólico Los Cocos; miembro fundador del grupo de innovación en la gestión de la construcción (GIGC) del INTEC. Ha escrito sobre temas de educación de la construcción y la construcción Lean, incluyendo para el Grupo Internacional para la Construcción Lean (IGLC).

\section{Fausto Fernely Moquete}

Es ingeniero civil dedicado a la ejecución y construcción de proyectos. Posee una maestría de administración de la construcción por el Instituto Tecnológico de Santo Domingo (INTEC).

Recibido: 22/09/2014 Aprobado: 23/02/2015 
